\title{
PROGRAMA PARA DESARROLLAR EL DESAPEGO EN LOS CUIDADORES DE PERROS GUÍAS
}

\section{Program aimed at developing emptional detachment in guide dogs' caretakers}

Michelle Juárez Maquet Makedonski. EAE Business School, España

Leandro Figueredo Alvarez. EAE Business School, España

Susan Puza Garcia. EAE Business School, España

Natalia Romero Guerrero. EAE Business School, España

David Ruiz Castillo. EAE Business School, España

\section{RESUMEN}

Con el presente proyecto de investigación para el desarrollo del desapego en los cuidadores de perros guía, queremos rendir homenaje a todos los cuidadores que de manera voluntaria, realizan una acción tan loable y necesaria para las personas invidentes. Hemos querido abordar el proceso de apego y desapego desde una perspectiva de regulación emocional diferente. A través de encuestas en profundidad a una muestra cualitativa con 3 cuidadores de perros guía que actualmente desempeñan esta función, nos adentramos en su vida cotidiana, conociendo de primera mano las situaciones, emociones y resistencias que encuentran en este proceso. Con la ayuda de referencias bibliográficas de personas psicoanalistas, psiquiatras, biólogos y profesionales en estas áreas, hemos podido evidenciar ciertos rasgos de comportamiento y estados emocionales que impactan en el vínculo afectivo de los seres humanos con los perros.

PALABRAS CLAVE: inteligencia emocional, apego, perros guías, mindfulness.

\section{ABSTRACT}

With the present research project for the development of detachment in the caregivers of guide dogs, we want to pay tribute to all the caregivers who voluntarily perform such a commendable and necessary action for blind people. We want to approach the process of attachment and detachment from a different emotional regulation perspective. Through in-depth interviews with a qualitative sample of 3 guide dog handlers who currently perform this function, we dive into their daily lives, knowing first-hand the situations, emotions and resistance they encounter in this process. With the help of bibliographic references from psychoanalysts, psychiatrists and professionals in these areas, we have been able to demonstrate certain behavioral traits and emotional states that impact on the affective bond of human beings with dogs.

KEYWORDS: emotional intelligence, attachment, guide dog, mindfulness. 


\section{Cómo citar el artículo:}

Juárez Maquet Makedonski, M., Figueredo Alvarez, L. Puza Garcia, S., Romero Guerrero, N. y Ruiz Castillo, D. (2020). Programa para desarrollar el desapego en los cuidadores de perros guías. Revista de Ciencias de la Comunicación e Información, 25(2), 133-155.

doi: http://doi.org/10.35742/rcci.2020.25(2).133-155

\section{PARTE INTRODUCTORIA}

\subsection{Introducción} - dijo el principito. ¿Qué significa "domesticar"?
- Es algo demasiado olvidado - dijo el zorro. - Significa "crear lazos..."
- ¿Crear lazos?

- Claro - dijo el zorro. - Todavía no eres para mí más que un niño parecido a otros cien mil niños. Y no te necesito. $Y$ tú tampoco me necesitas. No soy para ti más que un zorro parecido a otros cien mil zorros. Pero, si me domésticas, tendremos necesidad uno del otro. Tú serás para mí único en el mundo. Yo seré para ti único en el mundo...

Antoine de Saint-Exupéry

"El ser humano es un ser social por naturaleza", ya lo decía el filósofo Aristóteles (384-322 a.c) para señalar que desde que nacemos tenemos una característica social, en tanto que nuestra supervivencia depende del otro y que toda relación que construimos tiene un impacto en nosotros y en el ser con el que interactuamos, además de una incidencia directa en nuestra calidad de vida y bienestar.

Como lo estudiaremos a lo largo de este trabajo, en su teoría del apego, Bowlby (1999), manifiesta que el apego se presenta desde la infancia y es inherente a la relación humana para garantizar la supervivencia durante los primeros años de vida. Ahora bien, como lo plantea el Dalai Lama (2000) en su obra Samsara, como seres finitos y cambiantes, toda la existencia está sujeta a ciclos que intrínsecamente suponen un punto de partida o nacimiento, un desprendimiento o muerte, y un renacer, pero es precisamente allí, en ese proceso de generación de vínculos en las relaciones, y su posterior fase de desprendimiento o muerte, donde el ser humano queda expuesto a emociones de intenso dolor, pérdida y sufrimiento al no ser aceptado como un ciclo de la existencia misma.

Lo anterior, en occidente se conoce como el proceso de desapego, pérdida o duelo, y en la mayoría de los casos conlleva implícitas emociones, que como lo desarrollaremos más adelante, autores como Bowlby (1993) o Kübler (2016) las clasifican en fases, que consideran necesarias para dar paso a la aceptación y la creación de una nueva realidad.

Por lo anterior, contribuir a la gestión de las emociones que se presentan en el proceso de desapego entre cuidador y perro guía, al finalizar su etapa de convivencia, permite a nuestro equipo tomar una acción pertinente, a través de la creación de un programa basado en técnicas de mindfulness; orientado al acompañamiento y mitigación de los impactos dolorosos y traumáticos en los 
cuidadores de perros guías. Pues de lo contrario, de no ser atendidas y gestionadas dichas emociones, éstas deberán ser tratadas en psicoterapia, tal y como lo plantean los psicólogos Sharkin y Knox (2003), en su artículo: Pet loss: Issues and implications for the psychologist.

\subsection{Estado de la cuestión}

El siguiente trabajo de investigación está realizado para facilitar la gestión del desapego en el momento en que los cuidadores de los perros guías deben entregar al perro, después de culminar todo el periodo de entrenamiento y vivir con ellos toda la experiencia de su cuidado y aprendizaje especial durante un año completo. En ese sentido, el objetivo de este estudio es el siguiente:

Hemos detectado que hay una necesidad de acompañar al educador en el proceso de entrega del perro guía, después de un año de haber sido acogido en la casa de éste, debido al apego que se pudo haber generado en el tiempo que lo tuvo a cargo. Por esto, hemos visto oportuno crear un taller, que incluya también una guía práctica donde entreguemos diferentes estrategias para afrontar este momento de la entrega del perro guía y de esta manera, facilitar el proceso de desapego emocional. Esta necesidad identificada nos lleva a investigar más en profundidad sobre las teorías del apego y sus implicanciones.

El apego es una situación que se presenta desde la infancia, en donde uno de los involucrados representa una figura muy fuerte y/o sabia. Esta situación es especialmente evidente a temprana edad. Este comportamiento se considera propio de los seres humanos y se sabe que le acompañará desde el nacimiento hasta su muerte. El apego es más evidente cuando estamos niños, pero siempre está activo en toda nuestra vida (Bowlby, 1999).

Por su parte, y desde otro punto de vista, Goleman indica que "el apego determina las personas a las que apelamos en busca de ayuda y aquellas otras que más extrañamos cuando están ausentes" (2006, p. 193).

Por otro lado, según Walter Riso (2012, p. 81), el apego es una vinculación mental y emocional a objetos, personas, actividades, ideas o sentimientos, originada en la creencia irracional de que ese vínculo proveerá de manera única y permanente placer, seguridad o autorrealización.

En el II Análisis Científico del Vínculo entre las personas y los animales de compañía realizado por la Fundación Affinity (2014) se demostró que "un 31\% de los encuestados consideran que su animal de compañía es mucho más importante que cualquiera de sus amigos. El $75 \%$ de la población estudiada tiene un vínculo emocional muy intenso con su animal de compañía; es una fuente importante de apoyo emocional, sobre todo en situaciones difíciles". De allí la importancia de considerar un acompañamiento que sirva de apoyo a los cuidadores que han tenido a cargo a su perro guía, creando estos vínculos tan fuertes. 


\subsection{Antecedentes}

Plantea Gutiérrez (2007, p. 164) "Las relaciones entre hombres y animales han sido variadas y crecientes. A lo largo de la historia los animales han sido utilizados como medio de trabajo, como fuente de alimento, como medio de entretención, como protección para el hogar o el territorio, como símbolo o instrumento sagrado objeto de culto, como modelos de investigación biomédica y conductual, como guía para personas discapacitadas y como fuente de afecto para sus dueños.

Pero, ¿cómo se creó la relación entre una persona ciega y un perro? Veamos un poco de historia, de acuerdo a la investigación que realizó la Federación Internacional de Perros Guía (IGDF por sus siglas en inglés) y que aparece publicada por la Asociación de usuarios de perros guía de Murcia (2009).

- 1 Siglo A.C: primer registro en un mural donde se observa un humano invidente con un animal en el Herculaneum romano.

- 79 D.C: en Pompeya, se descubrió un mural de un individuo guiado por un perro.

- 1200 D.C: un pergamino chino, muestra a un individuo guiado por un perro. El pergamino ahora se encuentra en el Museo Metropolitan de Nueva York,

- 1260 D.C: una referencia irlandesa, que se atribuye a Bartholomew, aparece un perro guiando a un individuo.

- 1500-1700 aparecen referencias de perros guiando a individuos durante el siglo XVI en madera, grabados y pinturas en todo el mundo.

- 1715 "Blind Beggar of Bethnal Green" es una balada que cuenta que un caballero que perdió la vista en una batalla y por ese motivo se convirtió en un mendigo recibió por parte de su amigo un perro con correa y una campana.

- 1727 Gainsborough (1727-1788) pintó un cuadro llamado "Blind Man on the Bridge" en el que se muestra un perro guiando a su dueño.

- 1780 Se tiene referencia de la primera prueba de forma sistemática para adiestrar a perros con el fin de que ayudaran a personas ciegas. Ésta tuvo lugar, en 'Les Quinze-Vingts' un hospital para personas invidentes en París.

- 1819, Johann Wilhelm Klein, fundador del Instituto para la educación de los ciegos (Blinden-Erziehungs-Institut), en Viena, escribió un libro para enseñar a los ciegos las técnicas de adiestramiento de los perros guía, llamado "Lehrbuch zum Unterricht der Blinden".

\subsection{Descripción del problema}

Teniendo como premisa el estudio de la inteligencia emocional, podemos entender que en la relación humano - animal entre el cuidador y el perro guía, empieza a haber una relación muy profunda de complicidad y de emocionalidad.

Las entrevistas realizadas con los diferentes cuidadores de perros guías nos da una mirada profunda del lazo de apego que surgen en dicha relación. Hemos visto que en las interacciones, ninguno de los cuidadores quiere pensar en el momento que le digan que su perro es apto para guía y tengan que devolverlo. Nos comentan, que saben por otros cuidadores, que la experiencia de entregar al perro guía es muy 
dolorosa, por lo cual algunos optan por volver a adoptar varias veces para llenar el vacío que sienten, pero no encuentran ningún acompañamiento de parte de la institución para poder mitigar los sentimientos de poder desapegarse de su mejor amigo. Es por ello, que este "programa para el desarrollo del desapego en cuidadores de perros guía", apoyará a los posibles efectos que pasará el cuidador en el proceso de entrega del perro.

\subsection{Metodología de la investigación}

\subsubsection{Tipo de investigación}

En la realización del "programa para el desarrollo del desapego en cuidadores de perros guía" se utilizó un enfoque cualitativo, donde se realizaron tres encuestas a diferentes perfiles de cuidadores de perros guía.

Los resultados de la encuesta se orientaron a una investigación - acción - aplicada, porque diagnosticamos el problema, pero en esta oportunidad no lo aplicamos.

Para este trabajo, tuvimos la colaboración de tres personas que son actualmente cuidadores de perros guías y les hicimos una entrevista en profundidad, donde nos ayudó a identificar las oportunidades de indagación.

\subsubsection{Objetivo general}

Debido a los antecedentes que hemos estudiado y a las primeras conversaciones que tuvimos con algunos cuidadores de perros guía, el objetivo del presente trabajo es el siguiente:

Diseñar un programa que permita hacer frente al apego en los cuidadores de perros guía.

\subsubsection{Objetivos específicos}

Para lograr de manera óptima el diseño del programa que permita hacer frente al desapego en los cuidadores de perros guía, hemos definido cuatro objetivos específicos que abordaremos en el presente trabajo:

- Conocer las causas que generan el apego en la relación entre cuidador y perro guía.

- Identificar cómo gestionan el apego los cuidadores de perros guía.

- Elaborar un taller aplicativo que contribuya al proceso de desapego del cuidador con el perro guía.

- Realizar una guía práctica de soporte al taller que contribuya al proceso de desapego del cuidador con el perro guía. 


\section{PARTE GENERAL}

\section{Marco Teórico}

\subsection{Breve historia de la teoría del apego}

John Bowlby, psicólogo, psicoanalista, psiquiatra infanto juvenil, profesor, científico, autor, pensador y humanista; y gracias a su lucha incansable de más de 30 años para incorporar una nueva forma de abordar la psicología o psiquiatría infanto juvenil, instauró el término del apego, de hecho, se le conoce como "El padre de la teoría del apego". Al principio no fue aceptado por la "sociedad psicoanalítica británica", y fue rechazado rotundamente, porque su teoría difería completamente de las teorías iniciales, pero poco a poco y gracias a las contribuciones de otros investigadores especialistas en etología, pudo comprobar en el campo, los diferentes roles del apego. A través del diagnóstico que se realizaba a los niños, el psiquiatra decidió incluir tratar y estudiar a la madre. Fue su curiosidad por la etología, específicamente sobre la impronta de Konrad Lorenz, donde se muestra que de recién nacidas, las crías de las ocas siguen a cualquier objeto que se mueve en un espacio cercano. Y al cabo de un tiempo, las crías solo siguen, tan solo al objeto que le es familiar, sea la oca madre, otro animal o una persona, y lo hacen independientemente de si han recibido alimento o no, lo que motivó a Bowlby y se convirtió en una pieza fundamental para lograr acuñar la teoría del apego (Ezquerro, 2017).

Harry Harlow, prestigioso psicólogo y etólogo, amigo de Bowlby publicó: "La naturaleza del amor", basada en estudio de primates no humanos, donde se rechaza la idea de que el apego existe por solo gratificación de comida. Hizo unas pruebas con Macacos donde crearon dos madres inanimadas, y fueron colocadas delante de macacos bebés. Una madre inanimada estaba hecha de alambre y llevaba una botella con leche en los brazos a modo de biberón. La otra estaba hecha de un tejido suave parecido a la felpa y no ofrecía alimento. El descubrimiento fue que los macacos bebés prefirieron permanecer más tiempo junto a la madre de felpa, aunque esto significaba no tener alimento. El hallazgo no dejaba dudas de que los bebés macacos preferían un contacto de confort antes que la leche. Esa forma de estar les representaba una forma segura de estar. Si la madre responde de forma asertiva hacia el niño y le cría de forma cariñosa, cuando esté ausente es muy probable que satisfaga dicha necesidad y el niño pueda explorar el medio ambiente que le rodea. Una persona que ha recibido esos estímulos es muy probable que, en su vida adulta, pueda alejarse de la familia sin problema, pero siempre manteniendo contacto y volviendo tarde o temprano a su hogar. Por el contrario, la persona que no haya desarrollado ese vínculo, es probable que se sienta intensamente solo y sin arraigos en la vida adulta. Por ello, hemos querido revisar los inicios de Bowbly para sustentar nuestra hipótesis sobre los vínculos de los cuidadores de perros guías. En esta etapa el cuidador genera lazos fuertes durante un año de convivencia que luego es difícil romper (Ezquerro, 2017).

En el libro de John Bowlby, "El apego y la pérdida" (1998), hacen un experimento de observar, cuando la madre se aleja y observan el llanto del niño y el seguimiento de la madre cuando se aleja y el saludo y acercamiento cuando vuelve. Un niño cuando 
empieza a gatear, se permite explorar el espacio y lo recorre. De vez en cuando regresa a su madre para corroborar que esté ahí. En su búsqueda de nuevas cosas y la exploración que realiza, se ven interrumpidas cuando surge un momento de a) miedo o se hace daño y /o B) la madre se desaparece. Cuando suceden estos casos, el niño vuelve rápidamente a buscar a la madre. Esto sucede en la mayoría de casos en niños de 7 meses, sin embargo, en niños de 8 meses en adelante, les sucede a todos. Cuando la madre está presente, el niño siente confianza y ganas de explorar otros lugares. Caso contrario, cuando advierten la ausencia de la madre, se tornan tímidos y en algunos casos les da angustia.

Según Arturo Ezquerro (2017), el apego es a la vez un instinto y una relación, sus bases están cimentadas en la biología evolutiva y la etología. Sin embargo, los instintos no existen aisladamente, por ello Bowlby estudió a los pacientes y familias, de forma conjunta. El apego, también llamado "vínculos de base segura", y la calidad del cuidado no son atributos de la persona, se trata de constructos diádicos que se generan en los primeros meses de vida y que no están supeditados a la necesidad de alimentarse o necesidad sexual. El apego es considerado como una clase distinta a ello y no contiene nada infantil o patológico.

Bowlby (1995) hace un estudio del desarrollo de la conducta del apego de cinco especies de primates, que son: Monos Rhesus, mandriles, chimpancés, gorilas y el ser humano y en este estudio, también observa que existe un debilitamiento de la conducta del apego. A medida que los primates van creciendo surge una separación entre el cuidador y el niño, porque su entorno se vuelve muy relevante y pasan más tiempo con sus pares o los próximos adultos que serán. Cabe resaltar que no existe ninguna pauta de conducta donde estén involucrados tantos sentimientos, como lo es el apego. Las figuras que para el niño son importantes y corresponden a su conducta de apego, le generan amor y bienestar. Mientras el niño está en presencia de un cuidador que genere apego, él se sentirá mucho más seguro y confiado. El saber que puede perderla, le genera dolor, angustia y significa una amenaza para el niño.

\subsection{Diferencias entre apego y dependencia}

Existe una diferencia entre apego y dependencia, (Bowlby, 2006) mientras la dependencia no se halla relacionada para mantener proximidad, no está dirigida a una persona específica, no implica un vínculo o sentimiento intenso o duradero. Tampoco se le atribuye ninguna función biológica. Por otro lado, en las implicaciones de la dependencia existen valores que van en contraposición al concepto del apego. Mientras calificar a alguien que tiene dependencia emocional, se toma de forma negativa, hablar de que esa persona tiene apego, tiene una connotación positiva, más aún, una persona que es "desapegada", es vista de forma no muy favorable.

\subsubsection{Rasgos del apego}

Uno de nuestros objetivos del trabajo, es conocer cuáles son las causas que generan el apego entre la relación entre cuidado y perro guía, por ello es importante revisar los rasgos que presenta el apego según J. Bowlby (1995) y son las siguientes: 
- Especificidad: el apego es dirigido de forma específica, con un claro orden de preferencia

- Duración: es un hábito que permanece de forma constante y aunque se puedan llegar a trabajar, los apegos primitivos son muy persistentes y no pueden abandonarse fácilmente

- Intervención de emociones: muchas de las emociones aparecen de forma temprana y se van marcando conforme la formación de la persona. Se pueden experimentar situaciones de júbilo, felicidad cuando se experimenta la renovación de un vínculo, como enamorarse o mantener un vínculo con alguien y todo lo contrario si se experimenta una pérdida del vínculo afectivo, lo cual podría generar situaciones de ira, rabia o tristeza, o desconcierto cuando se pierde

- Ontogenia: es lo que sucede a los primeros nueve meses de vida a los bebés cuando lactan leche de su madre. Mientras más contacto tengan con la madre, más formarán el vínculo de apego. Mientras más saludable sea ese vínculo, se activará en el futuro mucho menos el sentimiento de apego, es decir, si existe una satisfacción de forma correcta en el bebé dentro de los 9 veces y ese sentimiento es atendido, por lo general cuando crezca no tendrá que activar apego en otras personas porque fue satisfecho de niño.

- Aprendizaje: es parte del desarrollo clave distinguir entre lo familiar y lo extraño. También se podría desarrollar el sentimiento de apego dentro del papel de castigos impartidos por el cuidador

- Organización: el apego inicial se forma de manera sencilla hasta el primer año de edad, luego de eso, este comportamiento se vuelve mucho más complejo, a base de sistemas comportamentales. Se activan por determinados estímulos del medio, como, por ejemplo: la fatiga, el hambre y cualquier cosa que nos asuste. Lo que pone fin a este miedo, es la figura estable de la madre

- Función biológica: el comportamiento del apego es posible en todos los mamíferos y una porción de ellos puede tener este apego en la vida adulta

\subsubsection{Tipos de apego}

Después de revisar los rasgos que presenta el apego, también es importante conocer los tipos de apego que puede presentar una persona para entender lo que puede suceder en la relación del cuidador y el perro guía que tiene a su cargo.

Según Mary Ainsworth, basándose en la teoría de Bowlby (1998), hay 3 niveles del apego:

- Apego Seguro: el cuidador es una base segura, es decir que la persona confía en él. Está presente cuando se necesita ayuda, en una situación de miedo o adversa. Transmite seguridad, confianza, protección y amor. Si el cuidador se presenta de este modo, es muy probable que el niño incorpore en su vida herramientas competentes y prácticas para afrontar adecuadamente las dificultades y obstáculos de la vida. La madre está comprometida con la educación de su hijo, es responsable y hay confianza.

- Apego Inseguro: en éste hay una falta de figuras adecuadas del apego. En este caso el cuidador tiene problemas de sintonizar, puede actuar de manera molesta y descalificadora, lo cual ocasionará que el niño se sienta inseguro y que experimente sentimientos que no pueda gestionar o modular como la 
rabia o la tristeza. Construye una identidad personal de una imagen de sí mismo no amable y merecedora de cuidados o incapaz de moverse en el mundo o de construir vínculos afectuosos o de confianza. Este tipo pueden ser agresivos, provocadores, cerrados o controladores.

El apego inseguro se subdivide en dos: apego evitativo y apego ambivalente y la explicación es la siguiente:

1. Apego Evitativo: siente angustia de perder a la persona que representa su base Segura, como puede ser una pareja. Se llama Evitativo porque, en lugar de tomar confianza, minimiza sus emociones para protegerse de un eventual sufrimiento. A este tipo de personas no les gusta hablar o en lugar de utilizar el lenguaje, utilizan el silencio o el enfado para comunicar lo que sienten. Se les complica amar o expresar lo que sienten porque sienten que deben cuidarse de una amenaza que no llega y no identifican.

2. Apego Ambivalente: que es cuando quiere pasar todo el tiempo con su pareja, incluso cuando la pareja sale con sus amistades, quieren estar presente. Se llama ambivalente, porque requiere estar presente todo el tiempo con la pareja y por otro lado, si les falla y no está presente cuando lo demanda, entonces hay emociones de enojo, ira o desprecio.

3. Apego Desorganizado: vemos a cuidadores que están asustados pero también son asustadores, lo cual crea en el niño miedo y dificultad de mantener todas las partes del yo, viven en una contradicción de la figura básica, la base segura, por un lado calman las angustias y por otro lado las incrementan, generando desarrollo autoevaluativo negativo, desprecio así mismo, comportamientos de desconfianza hacia los demás, en ocasiones estos niños no interactúan con sus iguales ni con sus cuidadores, al no contar con las habilidades y refuerzos necesarios, no saben cómo responder a los demás a diferentes situaciones.

\subsubsection{Niveles de apego}

Para comprender cuál es la carga emocional cuando uno cuida a un perro, tomamos estos diferentes niveles de apego que, según Miguel Ruíz (2014), existen cinco. M. Ruiz nos invita a un viaje, poniendo de ejemplo a una persona que le gusta el fútbol y como sus gustos se vuelven desde un gusto superficial hasta volverse en fanatismo, todo esto involucrando los diferentes niveles de apego. De igual forma dependiendo de cada persona en forma particular, es probable que por su forma de ser y de su personalidad, enfrenten los niveles de apego de una forma más llevadera para devolver al can (nivel uno, superficial) o de una forma con un apego más profundo que puede ocasionar sentimientos mucho más marcados de apego, donde le cueste desapegarse de forma correcta.

Los niveles del apego son los siguientes:

- El primer nivel, lo llama el yo verdadero: él dice que, si es una persona que ve el football y va a verlo sin importar ser de algún grupo en particular, simplemente le gusta verlo, participar y disfrutar sin estar al lado de ninguno; a este nivel disfruta del momento sin apegarse a él. Es usted controlando el conocimiento. Experimenta la forma más pura de gozo, la que surge de su puro deseo de experimentar la vida sin condiciones. 
- El segundo nivel es preferencia: aquí, la persona que acude a ver el football elige un bando y se dedica a alentar a ese, pero cuando termina se olvida de ello. A este nivel ha invertido una parte de sí, ha tenido una preferencia en un equipo. Esta capacidad de apegarse y desapegarse fácilmente le permite manifestar una parte emocional suya que disfruta con los altibajos de su equipo. La vida tiene lugar y usted la comparte con los que le rodean.

- El tercer nivel lo llama Identidad: en esta experiencia, se es fan de un equipo. Es su equipo favorito, lo vive, si pierde se va triste y si gana se va alegre. Si pierde acepta la derrota mientras felicita al equipo contrario. Su autoestima no se basa en el resultado. Sus sentimientos y emociones no condicionan cómo se relaciona con los demás o consigo mismo. A este nivel, el apego a su equipo empieza a afectar tu vida personal fuera del estadio al relacionarse con el mundo como un hincha. Su apego invade un mundo que nada tiene que ver con él.

- El cuarto nivel es la interiorización: su equipo de football se ha vuelto parte de su identidad. La historia de derrotas y victorias ahora trata de usted y le afecta su autoestima. Su equipo forma parte de usted y condiciona su identidad, por la creencia de lo que significa ser un "auténtico hincha". Cree que los que no piensen como usted, están equivocados. En este punto ha pasado de identificarse con la creencia a interiorizarla.

- El quinto nivel es fanatismo: su equipo es lo máximo y todos los demás son nada. Está súper identificado y si su equipo pierde, está mal todo el día. Ha adoptado la idea de que su equipo es lo mejor. Su creencia se vuelve más importante que la experiencia.

Estos niveles se pueden adaptar a la relación que el cuidador experimenta con su perro, que puede iniciar en un primer nivel y mantener esa experiencia grata sin condiciones o ir aumentando de nivel hasta sentir que su vínculo es más importante que la propia experiencia que vive con el.

\subsection{El duelo}

Como ya lo hemos visto en el capítulo anterior, y gracias a la Teoría de la vinculación, desarrollada por John Bowlby (1979-1988) sabemos que el ser humano, marcado por una infancia prolongada, es un ser social por naturaleza, que crea vínculos con otros para garantizar sus necesidades de supervivencia, así como el desarrollo de su identidad y su sentido de pertenencia. Y es precisamente, ante la ruptura o amenaza de ruptura de dichos vínculos, que se produce un estado afectivo de gran intensidad emocional al que llamamos duelo.

De acuerdo a lo que plantean Field, Gavish, Orsini y Packman, (2009), en términos de impacto psicológico, el proceso de duelo que se atraviesa tras la muerte 0 pérdida de una mascota es comparable al proceso de duelo que se vive tras una pérdida humana. Por lo anterior, abordaremos el duelo y sus efectos como objeto de estudio, para entender el proceso que pueden llegar a atravesar los cuidadores de perros guía, al momento de tener que regresar a su mascota para que continúe con su entrenamiento. 


\subsection{1. ¿Qué es el duelo?}

El duelo, del latín dolus: dolor, posee diferentes significados otorgados por la Real Academia de la Lengua:

- Dolor, lástima, aflicción o sentimiento

- Demostraciones que se hacen para manifestar el sentimiento que se tiene por la muerte de alguien

- Reunión de parientes, amigos o invitados que asisten a la casa mortuoria, a la conducción del cadáver al cementerio, o a los funerales

- Fatiga, trabajo.

Por su parte, Worden (2010), hace referencia en su obra en inglés a las palabras "mourning": proceso que se produce después de una pérdida y "grief": experiencia personal de la pérdida.

El psiquiatra e investigador, Doctor Javier García-Campayo (2012) en su libro"Manejo del duelo en atención primaria", se refiere al duelo como: "el conjunto de procesos psicológicos y psicosociales que siguen a la pérdida de una persona con la que el sujeto estaba vinculado afectivamente". Y presenta varios conceptos que se relacionan con dicho proceso, como son:

- Luto: que representa los aspectos sociales y antropológicos del duelo. Es un proceso psicológico, pero que tiene su expresión en las tradiciones y culturas, generalmente se ha asociado a signos externos (en occidente, por ejemplo, el uso de la ropa de color negro) que los familiares muestran durante el tiempo en que se supone se encuentran transitando el duelo..

- Sentimiento de pérdida: que hace referencia al estado afectivo que predomina durante el proceso de duelo.

- Elaboración del duelo: relacionado con la serie de procesos psicológicos que conllevan a la aceptación de la nueva realidad del sujeto.

El duelo es también uno de los objetos de estudio del padre del psicoanálisis, Sigmund Freud y en su publicación: Duelo y Melancolía, habla de dicho proceso como la reacción a la pérdida de un ser amado, o de una abstracción equivalente: la patria, la libertad, el ideal, etc. El neurólogo, crea un modelo inspirado en la depresión, en el que plantea una clara diferencia entre la persona que vive un proceso de duelo, y aquel a quien denomina como melancólico Freud (1917).

Además, insta a que jamás se debe considerar el duelo como un estado patológico y someter al sujeto a un tratamiento médico, aunque se trate de un estado que le impone considerables desviaciones de su conducta normal. Por su parte, indica que la melancolía, se caracteriza psíquicamente por un estado de ánimo profundamente doloroso, una cesación del interés por el mundo exterior, la pérdida de la capacidad de amar, la inhibición de todas las funciones y la disminución de amor propio. Esta última se traduce en reproches y acusaciones, en las que el paciente se hace objeto así mismo, y puede llegar incluso a una espera delirante de castigo (Freud, 1917).

Por su parte, el mismo Bowlby (1993) contempla el duelo como todos aquellos procesos psicológicos, conscientes e inconscientes, que la pérdida de una persona amada pone en marcha, independientemente del resultado. 
Y en una acepción más reciente, la psicoterapeuta Alba Payás (Puigarnau, 2010) define el proceso de duelo como la pérdida de relación, la pérdida del contacto con el otro, que rompe el contacto con uno mismo.

Pangrazzi (1993) plantea que el proceso de duelo es inherente a la existencia humana como la muerte en sí; no obstante, se hace evidente que dicho proceso está relacionado a la pérdida y no necesariamente a la muerte en su sentido más literal. En ese orden de ideas, podríamos decir que atravesamos diferentes procesos de duelo durante nuestra vida: ya sea la ruptura de una relación de pareja, una mudanza, un despido o cambio de trabajo, la pérdida de un órgano de nuestro cuerpo o el fallecimiento de un ser querido.

Así, ninguna persona puede decidir de forma consciente cómo reaccionar ante una pérdida, pues dicha reacción se verá influenciada tanto por factores internos como externos, pero de acuerdo a la intensidad del apego que se tenga hacia "lo perdido" se podrán identificar factores determinantes durante el proceso de elaboración del duelo (Worden, 2010)

\subsubsection{Factores que influyen durante el proceso de la elaboración del duelo}

Para entender mejor lo que puede influir en el proceso de duelo o pérdida, se detalla los factores presentados por el doctor W. Worden (2010):

- Quién era la persona fallecida: el parentesco, y el grado de cercanía determinarán el alcance de la pérdida.

- La naturaleza del apego (la intensidad del vínculo): la reacción emocional aumentará su gravedad proporcionalmente a la intensidad de la relación afectiva.

- Tipo de muerte: existe una tipología de la muerte catalogada bajo las categorías NASH: natural, accidental, suicidio y homicidio. Saber cómo murió la persona nos aportará información sobre cómo va a elaborar el duelo la persona superviviente.

- Antecedentes históricos: si ha tenido pérdidas anteriores y cómo se elaboraron dichos duelos. Es importante conocer la historia de salud mental previa de la persona, si tiene, o ha tenido, tendencia al abuso del alcohol o drogas u otros comportamientos adictivos o perjudiciales para su salud.

- Variables de la personalidad: Bowlby (1980, citado en Worden, 2010) incluye variables como la edad y el sexo de la persona, así como antecedentes de cómo ha manejado otras situaciones de crisis en su vida y su capacidad de resiliencia. También, se contempla que las creencias y valores de la persona pueden influir.

- Variables sociales: la subcultura étnica y social son sólo dos entre muchas. El grado de apoyo emocional y social percibido que se recibe de los demás, tanto dentro como fuera de la familia, es significativo. La mayoría de estudios encuentran que aquellos que progresan menos en el duelo tienen un apoyo social inadecuado o conflictivo.

- Tensiones presentes: cambios simultáneos y crisis que surgen después de la muerte, incluyendo graves cambios económicos. 


\subsubsection{Fases del proceso de duelo}

Para entender en qué fase del proceso de desapego se pueden encontrar los cuidadores de perros guía estudiaremos las fases del proceso de duelo, pues, aunque se haga evidente que las personas reaccionamos y expresamos de manera totalmente subjetiva nuestras pérdidas, y que hay factores determinantes que influyen la asunción del duelo, varios autores Bowlby (1961), Kübler (2016) han coincidido en que el proceso cuenta con distintas etapas que suelen ir acompañadas de una serie de emociones y sentimientos particulares.

Por su parte, Bowlby (1961), indicó que el duelo puede dividirse en tres fases principales, que posteriormente se ampliaron a cuatro

- Fase de embotamiento: es como un sentimiento de incredulidad. (Autores modernos como Kübler la denominan como fase de negación). Algunas personas pueden actuar como si nada hubiera sucedido y luego sorprenderse llorando. Este es un proceso que puede durar unos días o unas cuantas horas, donde se experimenta pena y dolor. El embotamiento es un mecanismo de defensa para abordar la información.

- Fase de anhelo y búsqueda de la figura perdida: durante esta fase la persona guarda la esperanza de que todo volverá a ser como antes. Hay una urgencia de reencontrarse con la figura perdida de buscarla. Durante esta fase, las personas pueden sufrir de ansiedad, insomnio, pensamientos obsesivos por la figura perdida, irritabilidad, agresividad e incluso puede llegarse al auto reproche.

- Fase de desorganización y desesperación: durante esta fase los sentimientos de profunda tristeza se desbordan. La persona siente el vacío y la soledad. El dolor por la pérdida se hace más evidente y se experimenta apatía, tristeza, desinterés.

- Fase de un grado mayor o menor de reorganización: finalmente la personas llega a aceptar que la pérdida es permanente. Hay una readaptación a la vida.

Para Bowlby (1961), el identificar estas fases no significa que exista un tiempo rígido para atravesarlas o que deban presentarse una después de la otra, ni siquiera que la persona deba experimentarlas todas. Sin embargo, con respecto al "doliente", conocerlas le permitirá establecer un esquema orientativo para determinar en qué fase del proceso se encuentra; y con respecto a su entorno (familia, amigos, colegas, etc) les permitirán mayor comprensión y empatía sobre el proceso de duelo que atraviese su ser querido o allegado. Ahora bien, aunque vivir el duelo en sociedad nos resulta positivo a la hora de legitimar nuestro dolor, cabe resaltar que también la pérdida es un proceso que se afronta desde la subjetividad y con recursos propios.

Lo anterior, nos resulta absolutamente pertinente para entender qué aspectos de las dimensiones humanas de los cuidadores de perros guía pueden estar siendo afectados durante el proceso de desapego, y nos resulta también útil para identificar las emociones que están surgiendo, entender los patrones de comportamiento que adoptan las personas, sus mecanismos de gestión y los riesgos a los que se ven expuestos física y psicosocialmente. 


\subsubsection{Los 6 tipos de duelo}

Worden (2010) clasifica el duelo en 6 tipologías, que veremos a continuación:

- Duelo anticipatorio: cuando se tiene conciencia de que se sufrirá una pérdida. Se diferencia de los otros porque los sentimientos suelen ser más ambivalentes e inestables, como la persona todavía está ahí, los dolientes alternan entre la cercanía y la distancia.

- Duelo ausente: quien se encuentra afectado bloquea sus sentimientos. En este caso opera un mecanismo de negación. Es tan fuerte el impacto que la persona no es capaz de afrontarlo. El problema es que el dolor oculto siempre retorna, ya sea como irritabilidad, ansiedad o de una enfermedad física.

- Duelo crónico: se presenta cuando una persona no logra superar la pérdida de un ser querido. Mantiene una postura de dolor. Las personas con depresión son más propensas a instalarse en este tipo de duelo, que también se convierte en un estilo de vida, prima la tristeza, la ansiedad y la culpa.

- Duelo retardado: aunque en un principio la persona intenta ignorar su dolor, pasado un tiempo emerge con gran fuerza y a veces en el momento menos esperado. También puede darse el caso de que una persona no puede experimentar el duelo en el momento en que se produce la pérdida.

- Duelo inhibido: es el tipo de duelo que experimentan las personas que tienen dificultad para expresar sentimientos. También se inhibe el duelo en personas con algún tipo de discapacidad cognitiva. O en situaciones en que el padre o la madre intentan mantenerse fuertes para no afectar a sus hijos.

- Duelo desautorizado: es el que se presenta cuando hay un rechazo del entorno hacia el dolor que experimenta una persona. Hay situaciones en las que desde un comienzo se desautoriza el dolor de una persona, como en el caso de la pérdida de un amante de una relación extramarital o el de una mascota.

En el caso de los procesos de duelo de las mascotas el valor de la pérdida no es reconocido o legitimado, pues generalmente se piensa que un animal de compañía es reemplazable (Wrobel y Dye 2003).

A efectos de este trabajo, entender el duelo como pérdida, en la cual el nivel de apego que tenemos con aquello que perdemos influencia nuestro proceso de gestión y superación del dolor, nos plantea una oportunidad en el abordaje del desapego como propuesta para mitigar los impactos dolorosos que tienen los cuidadores de perros a la hora de tener que entregar el perro que han cuidado.

\subsection{Bases neurobiológicas del apego}

Como se menciona en la primera parte de este capítulo, la teoría del Apego fue desarrollada por J. Bowlby, donde se puede identificar su influencia en la vida del ser humano y específicamente en sus relaciones afectivas, tomando como referencia el estudio del vínculo entre madre e hijo relacionado al instinto de supervivencia y que años después lo estudiaría además como producto de la actividad de una serie de sistemas de conducta, cuya consecuencia previsible es aproximarse a la madre (Bowlby, 1998). 
Con ello, nace la pregunta ¿qué sucede en el cerebro del ser humano que contribuye a crear estos vínculos de apego hacia otros? Para entender un poco mejor todo esto, se hace valioso mencionar los aportes de Jaak Panksepp (1998), famoso neurocientífico estadounidense quien estableció el término de "neurociencia de la afectividad" a la rama científica que estudia los mecanismos neurológicos de las emociones. Según Panksepp (1998), las emociones se forman a partir del funcionamiento de circuitos neuronales y sistemas bioquímicos específicos, los cuales determinan las tendencias de acción y de cuya interacción dinámica con los sistemas de representación del yo emergen todas las posibles experiencias subjetivas. En cuanto a la conducta maternal, Panksepp menciona que la madre desarrolla una conducta de protección hacia el bebé que radica en una transmisión hereditaria de signos biológicos a nivel especie, que va más allá del propio aprendizaje.

Los hallazgos neurobiológicos realizados en los últimos años con poderosas técnicas de neuroimagen refieren directamente a la implicación del sistema límbico en el proceso afectivo-emocional de creación de un vínculo en la mujer embarazada con el feto (Bartels y Zeki, 2004). Al momento del nacimiento, y estimulada por la succión durante el amamantamiento, una hormona llamada oxitocina se libera a la circulación sanguínea y aumenta sus concentraciones en el tejido cerebral de la madre, promoviendo el desarrollo de conductas de cuidado y la formación de un vínculo de apego hacia el recién nacido (Kendrick, 2000).

En ese sentido, es importante conocer un poco más sobre la oxitocina para entender sus efectos y su relación con el apego.

La oxitocina es un nonapéptido, sintetizado en el hipotálamo y almacenado en la neurohipófisis, cuya liberación es regulada por estímulos neurogénicos hormonales, humorales y emocionales. Ha sido tradicionalmente estudiada en relación a su papel promotor de las contracciones del útero en el parto y con el amamantamiento (Fernández et ál, 2018).

El Dr. José Luis Bonet (2016), menciona que se identifica un alto nivel de oxitocina durante todo el embarazo, el cual predice la calidad de conducta materna después del parto y esto es evidencia del impacto de la oxitocina en las interacciones sociales y los sentimientos de apego. Dentro de las acciones sociales de la oxitocina, se puede observar que produce un estado de preocupación, empatía y confianza hacia otros seres humanos.

Asimismo, Bonet (2016) explica la relación de los niveles de oxitocina con los sentimientos de apego materno-fetal durante el embarazo. Indica que cuando las madres observan las fotos de sus hijos, se activan las vías dopaminérgicas del circuito de recompensa en el cerebro de las madres, zonas que contienen altos niveles de receptores de oxitocina.

Por otro lado, el apego del niño se inicia desde el nacimiento cuando empieza a reconocer el sonido, olor y rostro de su madre así como el medio ambiente en donde se encuentra. El contacto inicial del infante con su madre activa las células del bulbo olfatorio que permite el reconocimiento de la madre a través del olor. Esta 
percepción de olor se convierte en aprendizaje gracias a la liberación de sustancias como la norepinefrina en el locus coeruleus, asociando el olor materno con su proximidad y cuidado para que finalmente con la repetición de esta conducta las vías que comunican con la amígdala impriman este condicionamiento (Insel y Young, 2001).

Después de entender cómo se relaciona la oxitocina con el apego entre madre e hijo en los seres humanos, ahora se describe el rol de la oxitocina en la formación de los vínculos de apego entre el ser humano y los animales, especialmente, con los perros cuyo tema está relacionado directamente con el presente trabajo.

El perro ha sido la primera especie domesticada por el hombre como resultado de un proceso interactivo de miles de años, el cual produjo relaciones de competencia, cooperación y coevolución. Durante este proceso, los perros han adquirido habilidades comunicativas que favorecieron su relación con los humanos, la cual representa una expresión de apego (Díaz Videla y López, 2017).

A esto se suma las consecuencias positivas para la salud asociadas con la interacción entre humanos y animales que pueden ser causadas por la liberación de oxitocina inducida por emociones positivas como el afecto y el amor y por la interacción física entre el ser humano y el animal (Uvnäs-Moberg, 1997). La interacción física entre humanos y perros involucra varios tipos de estimulación sensorial no nociva, como el tacto, la presión suave, el calor y los golpes, así como las señales olfativas, auditivas y visuales (Handlin et ál., 2011).

\subsection{Relación entre la inteligencia emocional y apego}

En los últimos años se ha resaltado de forma muy significativa la importancia de la inteligencia emocional y el impacto que esta tiene en las relaciones interpersonales y con nosotros mismos. Según define el autor Daniel Goleman (2012), la inteligencia emocional es una forma de interactuar con el mundo que tiene en cuenta los sentimientos y abarca habilidades como el control de los impulsos, la autoconciencia, la motivación, el entusiasmo, la perseverancia, la empatía y la agilidad mental y que además, configura rasgos del carácter como la autodisciplina, la compasión o el altruismo, que son esenciales para una buena y creativa adaptación social. Según el propio autor, hay cinco categorías de inteligencia emocional (Goleman, 2012):

1. Autoconciencia: la capacidad de reconocer una emoción tal como sucede. Ser conscientes de nuestras propias emociones, sus efectos sobre nosotros y la forma en que las expresamos, fortalecerá nuestra autoconfianza.

2. Autorregulación: el control o manejo de emociones desagradables como la ira, la ansiedad o la depresión permitirá reducir la intensidad y/o la duración de estas. También generamos autocontrol sobre reacciones impulsivas y comportamientos de abuso o dependencia. Aumentaremos nuestra adaptabilidad a diferentes entornos y estaremos más abiertos a nuevas ideas.

3. Motivación: motivarse para cualquier logro requiere objetivos claros y una actitud positiva. Si tenemos la capacidad de capturar pensamientos negativos a medida que ocurren, podemos reestructurarlos en términos más positivos, lo 
que nos ayudará a lograr nuestros objetivos. La motivación está hecha del impulso de logro, compromiso, iniciativa y optimismo.

4. Empatía: la capacidad de reconocer cómo se sienten las personas es importante para el éxito en nuestras relaciones personales y profesionales. Cuanto más hábiles seamos para discernir los sentimientos detrás de las señales de los demás, mejor podremos interactuar con ellos de acuerdo con las señales que envían.

5. Habilidades sociales: el desarrollo de buenas habilidades interpersonales casi que equivale al éxito en nuestra vida y en nuestra carrera. En nuestro nuevo mundo globalizado, todos tienen acceso inmediato al conocimiento técnico. Por lo tanto, el "don de gente" ahora es muy importante ya que nos permite comprender, empatizar y negociar mejor con los demás en una economía global. Esto no es solo necesario en nuestras relaciones laborales, sino que también nos trae muchos beneficios a nuestras relaciones personales y familiares.

Por otro lado, la relación existente entre el apego y la inteligencia emocional ha sido estudiada anteriormente. En el trabajo de Darío Páez et al. (2009) los autores se basan en la tipología de estilos de apego según dimensiones de Bartholomew (1990) expuestos en la Tabla 1 para mostrarnos la existencia de evidencias que sugieren una relación entre la inteligencia emocional y el estilo de apego, y cómo el apego seguro en adultos se asocia moderadamente a la claridad, capacidad de reparación, así como a la baja dificultad para identificar y describir las emociones.

Tabla 1: Estilos de apego según dimensiones de Bartholomew et al.

\begin{tabular}{|l|l|l|}
\hline & $\begin{array}{l}\text { Ansiedad ante el } \\
\text { Abandono (Baja) }\end{array}$ & $\begin{array}{l}\text { Ansiedad ante el Abandono } \\
\text { (Alta) }\end{array}$ \\
\hline $\begin{array}{l}\text { Evitación de intimidad } \\
\text { (Baja) }\end{array}$ & APEGO SEGURO & APEGO RECHAZANTE \\
\hline $\begin{array}{l}\text { Evitación de intimidad } \\
\text { (Alta) }\end{array}$ & APEGO EVITATIVO & APEGO TEMEROSO \\
\hline
\end{tabular}

Fuente: Adaptado de "Avoidance of intimacy: An attachment perspective", por Kim Bartholomew, 1990, Journal of Social and Personal Relationships 1990 7: 147, p. 163.

Agregan los autores además que se ha encontrado cómo los individuos adultos seguros son mejores interpretando emociones faciales negativas a diferencia de sujetos evitantes y que perciben mejor las emociones positivas que los sujetos ansiosos y cómo los individuos con otros tipos de apego le otorgan menos atención a los hechos emocionales. Estos tienen menos capacidad de regulación emocional y más dificultad para verbalizar emociones que los individuos con apego seguro (Darío Páez et ál, 2009).

La relación vista anteriormente entre la inteligencia emocional y el apego sugiere que los individuos con una inteligencia emocional plena están mejor capacitados para desprenderse emocionalmente y transitar de una forma más constructiva el proceso de desapego de su perro guía y en general. 


\section{PROGRAMA PARA EL DESARROLLO DEL DESAPEGO EN CUIDADORES DE PERROS GUÍA}

Este programa está diseñado para el desarrollo del desapego en cuidadores de perros guía. Consiste en un conjunto de acciones, prácticas mindfulness y aplicación de herramientas que se trabajarán con los participantes a través de un taller práctico. Asimismo, este taller será complementado por una guía práctica de soporte para mantener la continuidad de las acciones trabajadas.

\subsection{Objetivo de la implementación}

El objetivo general de la implementación de este este programa es dotar a los participantes de nuevos conocimientos y herramientas sencillas con soporte en la inteligencia emocional, y de esta manera les permitan hacer frente al apego que han desarrollado hacia el perro guía que han tenido bajo su cuidado en un determinado tiempo. Tanto las actividades desarrolladas en el taller como las píldoras mindfulness contenidas en la guía buscan ser un apoyo importante en el proceso de desapego que pueden practicar a diario sin necesidad de tener la guía de un especialista para realizarlo o iniciar con este proceso.

\subsection{Taller aplicativo}

Consideramos que para el programa sea más efectivo se requiere vivenciar de manera guiada los primeros inputs del mindfulness y de la inteligencia emocional, ya que no podemos asegurar que todas las personas están familiarizadas con estos temas. Una forma de vivenciar estos conceptos es a través del taller aplicativo que se describe a continuación.

\subsubsection{Descripción del taller aplicativo}

El taller consiste en un evento debidamente planificado para lograr transferir nuevos conocimientos, técnicas y herramientas a los participantes que estén dispuestos a hacer frente al apego generado hacia un perro guía que han cuidado personalmente. Este taller ofrecerá actividades que se desarrollarán con la dirección de quienes sustentan el presente trabajo, que harán a la vez de facilitadores de este taller soportados en sus conocimientos de inteligencia emocional y mindfulness así como técnicas claves de coaching.

Este taller tendrá una duración de 5 horas que se realizará jornadas divididas por bloques o módulos de acuerdo a lo que se coordine con la institución que lo solicite. Cada bloque incluirá una breve introducción y explicación de las habilidades a desarrollar, así como una actividad práctica que será dirigida por los facilitadores.

La primera parte del taller consistirá en una actividad de bienvenida y en trabajos prácticos para reconocer y aceptar las emociones suscitadas durante todo el proceso de cuidado al perro guía. En esta parte se dará inicio a las primeras prácticas de mindfulness a través de meditaciones cortas y guiadas.

En el segundo bloque se experimentará una actividad culmen para el desarrollo del desapego e interiorización de nuevas herramientas que ayuden a los participantes a 
poner en práctica cuando lo crean conveniente. Esta parte se finalizará con una práctica de agradecimiento y las conclusiones de todo lo aprendido.

\subsubsection{Objetivo del taller}

El objetivo del taller propuesto es experimentar de manera vivencial los beneficios del mindfulness e inteligencia emocional en el proceso de desarrollar el desapego en los cuidadores de perros guía, de manera que tengan un acompañamiento inicial en la introducción a estas prácticas. Por ello consideramos importante brindar el máximo detalle de cada una de las actividades a realizarse así como la duración y materiales de soporte requeridos.

\subsection{Guía práctica de soporte}

\subsubsection{Descripción de la guía práctica de soporte}

La guía práctica que se presenta es un complemento al taller aplicativo y consiste en un entregable escrito en el que se recoge los aspectos básicos y más importantes tratados en el taller, descritos de manera ordenada y concisa para que los participantes puedan acceder rápidamente a su revisión posterior. El contenido estará dividido en dos partes: el resumen estructurado del taller y píldoras de mindfulness de práctica diaria.

El resumen del taller incluirá la descripción de las teorías revisadas en cada bloque así como una explicación concisa de las actividades realizadas con cada una de ellas incluyendo el objetivo de las mismas.

Las píldoras mindfulness que se incluyen en la guía son pequeñas dosis de prácticas mindfulness descritas paso a paso de manera que el participante pueda recurrir a ellas en cualquier momento que lo requiera para continuar con el desarrollo del desapego. La duración de estas píldoras es de un máximo de 5 minutos y pueden realizarse en cualquier momento del día.

\section{CONCLUSIONES}

Luego de todo lo experimentado a lo largo de estos meses donde hemos podido interactuar con varios cuidadores de perros guía y luego de revisar varios estudios realizados sobre el vínculo humano-animal y la generación de apego en las personas, hemos podido concluir que:

- Si bien es cierto el apego condiciona el desenvolvimiento, éste no determina la evolución de la persona, porque se puede evolucionar y mejorar la comunicación y el sentido del relacionamiento: "Infancia no es destino".

- Contar con un programa de acompañamiento para hacer frente al proceso de apego que se genera en los cuidadores de perros guía, contribuye a que el proceso de entrega de su mascota sea menos doloroso, se transite de forma menos traumática y se obtenga un aprendizaje para la vida. 
Michelle Juárez Maquet Makedonski, Leandro Figueredo Alvarez, Susan Puza Garcia, Natalia Romero Guerrero y David Ruiz Castillo

- Comprender y compartir conocimiento acerca de las causas de apego que se generan en la relación humano - mascota, permite crear consciencia sobre el proceso que viven los cuidadores de perros guías al momento de devolver a su amigo para que continúe con su entrenamiento.

- Identificar las emociones, pensamientos, sentimientos y conductas que se manifiestan durante un proceso de desapego entre cuidador y mascota, le permite a éste conocerse, entender el proceso al que se enfrente y tomar las medidas necesarias para transitar el apego.

- Contar con una guía práctica de soporte, permite al cuidador tener a la mano herramientas de auto gestión aplicables para cada una de las fases de su proceso de desapego.

\section{Bibliografía}

Asociación de perros guías de Murcia. (2009). Historia del perro guía. https://perrosguiamurcia.org/nuestros-perros/historia-del-perro-guia

Bartels, A., Zeki, S. (2004). The neural correlates of maternal and romantic love. Neurolmage, 21(3), 1155-1166. https://doi.org/10.1016/j.neuroimage.2003.11.003

Bartholomew, K. (1990). Avoidance of Intimacy: An Attachment Perspective. In Journal of Social and Personal Relationships, 7(2), 147-178. https://doi.org/10.1177/0265407590072001

Bonet, J.L. (2016). Cerebro, emociones y estrés. Ediciones B Argentina S.A.

Bowlby, J. (1993). La pérdida afectiva. Paidós.

Bowlby, J. (1995). Una base segura: aplicaciones clínicas de una teoría del apego. Oniro.

Bowlby, J. (1998). El apego y la pérdida. Paidós lbérica Ediciones S A.

Bowlby, J. (1999). Vínculos afectivos: formación, desarrollo y pérdida. Ediciones Morata.

Campayo, J. G. y De Marzo, M. (2020). Mindfulness y Compasión, la nueva revolución. Siglantana.

Campos, F. (2012). Apuntes personales de la asignatura: Intervención en Crisis. Grado de Trabajo Social (3o). Curso 2012-2013. Universidad de Las Islas Baleares.

Páez, D., Campos M., Zubieta, E. y Casullo, M. (2009). Vínculo de Apego e Inteligencia Emocional: estado de la cuestión y el instrumento de Medición de M.M. Casullo. Fundación Marcelino Botín. 
Diario Veterinario. (2019, 25 de marzo). El 40\% de los hogares españoles cuenta con una mascota. Recuperado de https://www.diarioveterinario.com/t/1364780/40hogares-espanoles-cuenta-mascota

Díaz Videla, M. y López, P.A. (2017). La oxitocina en el vínculo humano-perro: Revisión bibliográfica y análisis de futuras áreas de investigación. Interdiscilinaria, 34(1), 73-90. https://doi.org/10.16888/interd.2017.34.1.5

Ezquerro, A. (2017). Relatos de apego: encuentros con John Bowlby. Psimática.

Fatjó, J. y Calvo, P. (2014). Whitepaper del II Análisis Científico del vínculo entre personas y animales 2014. Fundación Affinity. Recuperado de https://www.fundacion-affinity.org/observatorio/whitepaper-del-ii-analisis-cientificodel-vinculo-entre-personas-y-animales-2014

Fernández P. L., Moreno, A., Leza, J.C., Lizasoain, I., Moro, M., Portolés, A. (2018). Velázquez. Farmacología Básica y Clínica (19a edición). Editorial Médica Panamericana.

Field, N.P., Gavish, R., Orsini, L. y Packman, W. (2009). Role of attachment in response to pet loss. Death Studies, 33(4), 334- 355

Freud, S. (1917). Duelo y Melancolía. Academia.edu. https://www.academia.edu/3254913/Duelo y melancol\%C3\%ADa Sigmund Freu $\underline{\mathrm{d}}$

Fromm, E. (1975). Anatomía de la destructividad humana (19ª edición). Siglo XXI

Fundación Affinity y la Cátedra Fundación Affinity Animales y Salud del departamento de psiquiatría de la Universidad Autónoma de Barcelona. (s.f.). Estudio Juntos en casa. Consultado el 1 de julio de 2020. https://www.fundacionaffinity.org/perros-gatos-y-personas/infografia-estudio-juntos-en-casa

Goleman, D. (2012). Inteligencia emocional. Editorial Kairós.

Gutiérrez, G., Granados, D. y Piar, N. (2007). Interacciones humano-animal: características e implicaciones para el bienestar de los humanos. Revista Colombiana de Psicología, 16, 163-184. Recuperado de https://www.redalyc.org/articulo.oa?id=80401612

Handlin, L., Hydbring-Sandberg, E., Nilsson, A., Ejdebäck, M., Jansson, A. y UvnäsMoberg, K. (2011). Short-Term Interaction between Dogs and Their Owners: Effects on Oxytocin, Cortisol, Insulin and Heart Rate - An Exploratory Study. Anthrozoös, 24(3), 301-315. https://doi.org/10.2752/175303711X13045914865385

Herzog, H. (2012). Los amamos, los odiamos y los comemos. Kairós.

Herzog, H. (2014). Biology, culture, and the origins of pet-keeping. Animal Behavior and Cognition, 1(3), 296-308. https://doi.org/10.12966/abc.08.06.2014 
Michelle Juárez Maquet Makedonski, Leandro Figueredo Alvarez, Susan Puza Garcia, Natalia Romero Guerrero y David Ruiz Castillo

Insel, T., Young, L. (2001). The neurobiology of attachment. Nature Reviews Neuroscience, 2, 129-136. https://doi.org/10.1038/35053579

Katcher, A. y Beck A. (1993), Los animales de compañía en nuestra vida: nuevas perspectivas. Fundación Purina

Kellert, S. y Wilson, E. (1993). The Biophilia Hypothesis. Island Press.

Kendrick, K.M. (2000), Oxytocin, motherhood and bonding. Experimental Physiology, 85, 111-124. https://doi:10.1111/i.1469-445X.2000.tb00014.x

Kübler, E (2016). Sobre el duelo y el dolor. Editorial Luciérnaga

Lorenz, K. (2017). Hablaba con las bestias, los peces y los pájaros. Tusquets Editores S.A.

Lorenz, K. (2018). Cuando el hombre encontró al perro. Tusquets Editores S.A.

Maura, M. (2015). Duelo y Apego: De la creación del vínculo a la pérdida del mismo [tesis de grado, Universidad de les Illes Balears]. UIB repositori. Recuperado de https://dspace.uib.es/xmlui

Panksepp, J. (1998). Affective Neuroscience. Oxford University Press

Puigarnau, A. P. (2010). Las tareas del duelo. Ediciones Paidós.

Pangrazzi, A. (1993). La pérdida de un ser querido. Un viaje dentro de la vida.

Riso, W. (2013). Desapegarse sin anestesia. Grupo Planeta Spain.

Ruiz, M. (2014). Cinco niveles del apego, Los: Sabiduría tolteca para el mundo moderno (Crecimiento personal).

Sharkin, B. S., \& Knox, D. (2003). Pet loss: Issues and implications for the psychologist. Professional Psychology: Research and Practice, 34(4), 414-421. https://doi.org/10.1037/0735-7028.34.4.414

Tizon, J. L. (2004). Pérdida, pena, duelo: vivencias, investigación y asistencia. Grupo Planeta (GBS).

Uvnäs-Moberg K. (1997). Physiological and endocrine effects of social contact. Annals of the New York Academy of Sciences, 807, 146-163. https://doi.org/10.1111/j.1749-6632.1997.tb51917.x

Villar, A. A., \& Lorenz, K. (1974). El comportamiento animal y humano. In Revista española de la opinión pública (35), 400). https://doi.org/10.2307/40182195

William Worden, J. (2010). El tratamiento del duelo: Asesoramiento psicológico y terapia. Paidós Ibérica. 
Zapiain, J. G. (2009). Apego y sexualidad: Entre el vínculo afectivo y el deseo sexual. Alianza Editorial S.A.

Wilson, E. (1984). Biophilia. Harvard University Press

Wrobel, T.A. y Dye, A. (2003). Grieving pet death: normative, gender and attachment issues. Omega, 47(4), 385- 393. https://doi.org/10.2190/QYV5-LLJ1-T043-U0F9

\section{AUTORES}

Michelle Juárez Maquet Makedonski

EAE Business School, España.

Leandro Figueredo Alvarez

EAE Business School, España.

Susan Puza García

EAE Business School, España.

Natalia Romero Guerrero

EAE Business School, España.

David Ruiz Castillo

EAE Business School, España. 